\title{
L'accouchement dans le Valais central de 1850 à 1950
}

\author{
Par Rose-Claire Schüle
}

Le dialectologue qui entreprend des enquêtes dans le domaine francoprovençal du Valais central recueille, à côté des mots qui ne posent aucun problème, quelques termes qui sont de véritables énigmes, car leur origine est parfois difficile à découvrir, leur signification incertaine dans son évolution. C'est un de ces cas qui m'a amené à faire des recherches ethno-linguistiques autour de l'accouchement.

La parturiente et, dans certains endroits, l'accouchée, ou le temps des couches, portent le nom de palyoûla (ou une variante phonétique locale du même mot). L'aire de palyoûla, mot qui dérive indubitablement du latin palea "paille, son"1, s'étend du Queyras - à travers les Préalpes vaudoises, le Valais et l'Italie du nord $^{2}$ - jusque dans les parlers réto-romans des Grisons. Le mot n'est donc pas exclusivement valaisan; c'est une appellation propre aux régions alpines. En effet, les patois alémaniques de l'Oberland bernois, du Valais et des Grisons connaissent, eux aussi, des expressions semblables du type tomber dans la paille signifiant 'accoucher'. Ces expressions sont anciennes, car nous en connaissons des attestations en ancien provençal, c.-à-d. du temps des troubadours.

Quelle idée expriment-elles exactement? Cette image de la paille nous fait penser au contenu de la paillasse, aïeule de nos matelas. 'Tomber dans la paille' n'est-ce qu'un synonyme de 'se coucher dans un lit'? Mais alors pourquoi ce mot est-il confiné à la région alpine, moins riche en paille que les vastes plaines à blé ? Une enquête s'imposait.

Inutile d'insister sur le fait que des précisions ne s'obtiennent pas facilement dans un domaine aussi fortement taboué que celui de la procréation et de la naissance. Bien que nos investigations, menées avec prudence et avec patience, se soient étendues sur plus de trente ans, ce que nous publions aujourd'hui ressemble fort à une de ces céramiques anciennes qu'on a reconstituées à partir d'innombrables fragments et de tessons, qui ne donnent donc qu'une idée approximative de l'ensemble auquel ont appartenu ces morceaux. La majorité de nos renseignements proviennent de la commune de Nendaz (Valais), d'autres des districts de Sierre, de Sion et d'Hérens; des compléments proviennent de pointages faits dans toute l'aire de palyoûla.

Jusque dans l'immédiat après-guerre, vers 1948, il était exceptionnel qu'une Valaisanne des villages de montagne se rende à l'hôpital ou à la maternité pour un accouchement. Seuls les accouchements à domicile pouvant présenter un intérêt pour nous, la période pour laquelle nous avons cherché des renseignements 
couvre donc environ les années de 1850 à 1950 vu que la mémoire humaine ne transmet que les souvenirs d'une ou de deux générations précédentes.

Jadis les femmes accouchaient aussi bien aux mayens, à l'alpage que dans la maison principale du village. Autant dire qu'il leur était difficile de s'isoler: à l'approche de la naissance, leur premier soucis était d'éloigner les enfants aînés. On les envoyait chez les grands-parents, chez les tantes ou même chez des voisines, selon les convenances et les possibilités. Un enfant parmi les plus grands, le père ou un voisin allait chercher la sage-femme. Celle-ci, souvent avertie trop tard, arrivait, surtout chez les pluripares, après la naissance du bébé; c'est que le trajet à accomplir, à pied ou à dos de mulet, était long. Une voisine, une parente ou même le père avait rempli entre-temps et tant bien que mal l'office de la sage-femme.

Les sages-femmes que nous avons connues nous-mêmes dans les villages étaient toutes diplomées. Elles ne se distinguaient en rien de leurs collègues citadines, modernes, si ce n'est par une certaine habileté à pallier l'indigence d'hygiène et de sécurité.

La situation était bien différente au siècle dernier. Dans beaucoup de villages fonctionnaient encore des marraines, c'est-à-dire des «matrones», femmes désignées à l'office de sage-femme par l'assemblée des femmes mariées. Les «marraines» ne suivaient pas de cours, elles apprenaient ce que le médecin ou la sage-femme appelés dans les cas difficiles ou désespérés leur enseignaient. Les femmes choisissaient la «marraine» en fonction de la sympathie qu'elle leur inspirait, de la confiance qu'on pouvait avoir en elle, de son entregent et de sa situation financière aisée. En effet, on ne voulait pas grever de cette fonction une pauvresse surchargée de travail et d'enfants en bas âge. Il fallait que la «marraine» pût abandonner sa famille et son ménage à n'importe quelle heure et pour un temps indéterminé.

La «marraine» avait généralement elle-même des enfants et, de ce fait, avait une expérience personnelle. Elle avait souvent eu l'occasion d'acquérir un certain savoir-faire au contact d'une proche parente, sa mère ou sa grand'mère, qui officiait comme accoucheuse. Certaines d'entre elles semblent d'ailleurs avoir été fort expertes. Ces femmes étaient fréquemment choisies dans des familles où un ou plusieurs hommes faisaient office de rebouteux ou de guérisseurs du bétail. On attribuait volontiers à ces familles une faculté ou une prédisposition à l'exercice de la médecine; on pensait qu'elles détenaient un savoir traditionnel transmis de génération en génération.

Autrefois, lorsque la sage-femme arrivait chez une parturiente, elle s'assurait d'abord de l'état de celle-ci et de l'avancement du travail. Elle n'hésitait pas à 
procéder à une visite gynécologique approfondie, moins pour se rendre compte du degré de dilatation que pour «oindre». ${ }^{3}$ Sans désinfection aucune, sans même se laver les mains, la matrone s'enduisait les mains de la matière lubrifiante choisie (beurre frais ou cuit, huile de noix ou de marmotte, saindoux), puis elle faisait subir le même traitement au vagin et au col de la matrice de la parturiente. Pour rendre le "passage» plus aisé, elle administrait de la crème ou de la graisse de marmotte per os et frictionnait le nombril ou tout le ventre de la même matière grasse, pour «assouplir». Il n'était pas rare que la «marraine» apportait dans sa sacoche, et la matière lubrifiante, et la bouteille de vin ou d'eau-de-vie destinée à soutenir la femme lorsque la parturition était longue et pénible.

Si la future mère ou son mari ne l'avait pas encore fait, la «marraine» étendait une couche de paille de seigle propre, soit sur la paillasse ou le matelas (là où l'on connaissait ce luxe), soit sur le sol de la chambre commune, près du fourneau ou du banc selon les convenances familiales. Dans de nombreux hameaux et villages de montagne, les femmes accouchaient, comme nous allons le voir, sur cette paille fraîche; ailleurs on couvrait la paille de vieux journaux ou d'un drap. On n'utilisait jamais de draps propres, ni pour l'accouchement, ni pour la période des couches. Parfois on mettait des vieux journaux sur le drap pour le préserver de souillures.

Ensuite, après avoir mis cuire de l'eau et terminé tous les préparatifs, la «marraine» s'occupait de nouveau de la femme. Certaines «marraines» apportaient à leurs clientes des amulettes, surtout des médailles bénites de Sainte Anne. Elles allumaient un cierge de Sainte Agathe et veillaient à ce que la parturiente ait un chapelet bénit dans les mains. Les ceintures de Notre-Dame ${ }^{4}$ ne semblent pas avoir été connues dans le Valais central, mais çà et là, on mettait une rose de Jéricho ${ }^{5}$ dans un récipient d'eau. La femme ne se déshabillait pas complètement et certaines accoucheuses excellaient à travailler «à couvert» pour ménager la pudeur de la parturiente, mais elles s'assuraient au moins qu'aucune ceinture ou fermeture de l'habillement de la femme en travail ne fût fermée, afin de favoriser par magie sympathique l'ouverture du passage.

Quelle position adoptait-on généralement dans le Valais central pour l'accouchement? Au cours des années 1900 à 1950, on semble avoir accouché surtout en position couchée, sur le dos, soit dans le lit, soit sur la paille répandue au sol. Dans le premier cas, les genoux étaient surélevés par des coussins ou des bottes de paille et les pieds appuyés contre le bois du lit dès la fin de la période de dilatation; pour l'expulsion la femme se plaçait souvent en travers du lit, les jambes pendantes. Les femmes qui accouchaient au sol appuyaient les pieds contre la paroi de la chambre, la «marraine» ou une aide soutenant leurs genoux. 
Plusieurs informatrices âgées nous ont indiqué que leur mère ou leur grand' mère avait accouché à genoux, sur une couche de paille étendue sur le plancher de la chambre. La femme en travail se soutenait des coudes et des avant-bras sur le banc de la chambre, la matrone recevait l'enfant en se plaçant derrière la femme. Nos informatrices elles-mêmes ont accouché étendues, mais elles s'agenouillaient, par moment, lorsque «ça n'avançait pas». L'une d'entre elles a mis son troisième enfant au monde agenouillée comme sa mère. Elles ont toutes conseillé à leurs propres filles d'avoir recours à cette position en cas de difficulté. Ces femmes de la génération suivante (nous avons pu obtenir ce renseignement de deux d'entre elles) nous ont dit avoir expérimenté ce procédé, mais l'avoir abandonné cédant aux instances de la sage-femme. Elles n'ont plus mis de la paille devant le banc, mais des journaux et des linges.

Nos informatrices les plus âgées savaient toutes, par ouï-dire, que dans deux hameaux de leur commune, les femmes accouchaient assises sur l'angle de deux bancs croisés, face à l'ouverture de cet angle aigu. L'enquête dans ces deux hameaux fut délicate et difficile: comme nous l'avions déjà vécu lors d'autres investigations, un sentiment suraigu de pudeur et un certain déplacement des domaines taboués par rapport aux habitudes des autres villages de la commune ne permettaient qu'une conversation fort prudente sur le sujet. Une seule femme nous a parlé d'une «chaise spéciale» utilisée lors des parturitions. Elle-même ne l'avait ni utilisée ni vue, bien que, de son temps, la chaise obstétricale fît encore le tour du hameau. Le lien entre les deux versions nous fut fourni spontanément et involontairement. Lors d'une discussion sur un tout autre sujet et dans un autre village, la conversation roula sur la naïveté des habitants des deux hameaux précités qui sont considérés comme les Abdérites de la commune et à qui on attribue toutes les sottises possibles et impossibles. Notre interlocutrice, âgée, fille de «marraine», nous dit, pour illustrer le prétendu retard intellectuel des habitants de ces hameaux, que sa mère avait dû s'y rendre, à la fin du siècle passé, pour assister une femme en travail. Or, disait-elle, les femmes de C. étaient habituées à accoucher sur une chaise spéciale, et cette dernière s'étant cassée, elles refusaient d'accoucher à genoux ou sur le lit; elles préféraient rapprocher deux bancs et appuyer de manière instable leurs fesses au fond de l'angle ainsi formé, sans même avoir d'accoudoirs où se cramponner. Nous n'avons obtenu aucun autre détail sur cette chaise obstétricale et nous n'avons pu savoir si la «marraine» avait accouché sa cliente de cette manière ou non.

Enfin, nous avons appris qu'une femme de cette commune, mais d'origine haut-valaisanne, accouchait toujours debout en s'accrochant des deux mains à une boucle de corde attachée à la poutre maîtresse du plafond de la chambre 
commune. Cette femme aurait eu ses enfants avant la première guerre mondiale. Peu d'informatrices du village connaissaient cette position et plusieurs d'entre elles n'en avaient même jamais entendu parler, ce qui n'est pas fait pour étonner vu la complexité des relations sociales dans nos grandes communes valaisannes et le caractère intime et taboué du sujet.

Les résultats de nos investigations sur la position de la parturiente dans d'autres localités du Valais central concordent avec ceux de Nendaz: on accouchait un peu partout sur de la paille, couchée sur le lit ou sur le sol, à genoux ou parfois accroupie, assise et plus rarement debout. Les rares renseignements trouvés dans la littérature attestent l'accouchement en position debout notamment pour le Lœtschental ${ }^{6}$, mais également dans le canton de Glaris ${ }^{7}$ et dans diverses régions françaises ${ }^{8}$. La chaise obstétricale est signalée dans les Grisons ${ }^{9}$ et dans le canton de Vaud ${ }^{10}$. Nous n'avons pas trouvé d'autres attestation pour la position accroupie ou celle à genoux. Lorsque un accouchement est décrit sans que la position soit mentionnée, nous ne pensons pas nous tromper en admettant qu'il s'agit de celle, habituelle, couchée sur le dos.

Revenons à l'accouchement proprement dit. Il était fort rare qu'une femme restât couchée durant tout le temps des contractions de dilatation. En général, elle se déplaçait à travers la chambre, encouragée par la sage-femme à se mouvoir. Lorsque les contractions duraient des heures et en cas d'atonie, la «marraine» faisait absorber des infusions d'ergot de seigle ou de safran, du vin chaud, de l'absinthe fraîche ou sèche, hachée et pétrie dans du beurre, de l'eau-de-vie. Nous n'avons pas pu établir de relation entre l'administration de l'ergot au début ou au cours de la parturition et les décès en couches, fort rares d'ailleurs. Certaines sages-femmes n'avaient recours aux préparations d'ergot que post partem, pour hâter l'expulsion du placenta ${ }^{11}$.

Les «marraines» soutenaient les femmes fatiguées, par des massages dans la région des reins, par l'application de bouillottes chaudes dans le dos et entre les cuisses. Lorsque le col de la matrice ne se dilatait qu'imparfaitement, certaines accoucheuses entreprenaient soigneusement la dilatation, avec les mains nues, non désinfectées mais lubrifiées; cela, au dire des informatrices qui ont vécu cette intervention, avec un succès indéniable et rapide. Nul besoin de dire que les sages-femmes diplomées, elles, ont recours au médecin.

Ce que les femmes, comme les «marraines» et les sages-femmes, redoutaient le plus était une mauvaise présentation de l'enfant. En effet, lorsque l'enfant se présentait «en double» (on appelait ainsi la présentation du siège), «en travers» (du dos ou de l'épaule), la sage-femme ou «marraine» tentait une version en repoussant le bébé mais lorsqu'il se présentait des pieds ou d'un pied elles hési- 
taient à faire cette intervention ${ }^{12}$; selon la croyance populaire, cette présentation laisserait peu de chance à l'enfant de survivre. Dans plusieurs communes, on pensait d'ailleurs qu'un enfant né ainsi serait affligé du «mauvais œil», qu'il deviendrait sorcier ou, pour le moins, mauvais chrétien. C'est un des cas où l'on se résignait à faire appel au médecin afin qu'il "prenne l'enfant avec les fers», c'est-à-dire au forceps.

La dernière phase, celle de l'expulsion, se déroulait toujours dans la position choisie par la femme. L'assistante l'encourageait de collaborer avec la nature. Toutes nos informatrices, âgées ou non, étaient d'avis que la collaboration active et intelligente de la femme est le meilleur moyen de hâter la naissance tout en causant le moins de douleurs. Il faut remarquer que la Valaisanne éprouve peu d'appréhension et ne se plaint guère lorsqu'une naissance se déroule normalement. On méprise la femme qui s'est anormalement débattue et qui a crié pendant la naissance. Il se peut que quelques femmes accouchaient presque sans s'en apercevoir, c'est-à-dire facilement. Mais, comme le signale Müller pour le Lœtschental, on pensait dans le Valais central, et dans la région alpine en général, qu'une femme crispée et qui hurle pendant un accouchement sans complication démontre ainsi un manque de foi et de confiance en Dieu. A une époque où l'on ne pouvait pas encore faire appel au médecin et où l'on ne pratiquait pas les césariennes aussi couramment que de nos jours, cette foi a permis à certaines femmes de supporter sans se révolter ou sans trahir leurs douleurs des accouchements au forceps, sans anesthésiants. Les déchirures fort fréquentes étaient acceptées comme inévitables. Ce n'est que depuis la guerre qu'on fait appel au médecin, post partem, pour les soutures. Auparavant on laissait agir la nature, d'où une fréquence de prolaps de matrice et de complications qu'on a peine à s'imaginer aujourd'hui.

Lorsque la sage-femme a constaté une dilatation suffisante et surtout lorsque la femme indique que «le mal a changé», l'accoucheuse expérimentée exerçait une légère pression sur l'abdomen de la parturiente, tandis que des aides soutenaient, s'il était besoin, les genoux ou le dos de la femme, selon la position adoptée. Aussitôt que la tête apparaissait et que la reprise des contractions annonçait la naissance imminente, la sage-femme passait les doigts sous le menton de l'enfant pour dégager la tête, puis elle déplaçait le corps pour libérer les épaules. L'enfant arrivé au monde, la matrone ou une femme expérimentée s'en occupait. Quant à la femme, elle s'installait sur une seille, de préférence une seille à douve prolongée où elle pouvait se tenir comme à une poignée, pour l'expulsion du placenta. Si cette expulsion se faisait par trop attendre, on administrait de l'ergot et l'on donnait à la femme une bouteille vide dans laquelle elle devait souffler de 
toutes ses forces. Si rien n'y faisait, l'accoucheuse intervenait manuellement ou on appelait le médecin.

Je passe sur les détails d'intervention en cas d'hémorrhagie, sur les complications faisant suite à la naissance, sur les significations superstitieuses de la date et du moment de la naissance pour en venir aux conclusions.

Le mot de palyoûla qui nous a incité à cette recherche est donc le reflet de situations d'accouchement où la paille joue un rôle important, procédés qui semblent avoir été propres à la région alpine. Cette paille ne fut d'ailleurs pas brûlée, mais on la laissait se décomposer, quelque part à l'écart, sous une couche de branches de sapin, comme on le faisait pour le contenu des paillasses après les décès. Des recherches et des enquêtes plus étendues dans l'espace et dans le temps permettraient probablement de constater, comme il en est dans de nombreux autres cas, que cette zone alpine est une zone marginale où certains procédés (et certains mots) d'autrefois ont survécu. Les innovations venant des grandes villes et des plaines, ou, dans notre cas, des écoles de médecine, n’ont pénétré que peu à peu dans les Alpes. Le cas de la chaise obstétricale semble prouver que ce meuble a été introduit par des médecins ou pour le moins par des sages-femmes ayant passé par une école. Le fait qu'on n'utilise pour les accouchements que de la paille de seigle, jamais de la paille de froment, parle par contre pour l'origine autochtone du procédé.

\section{Notes}

${ }^{1}$ Cf. W. von Wartburg, Französisches etymologisches Wörterbuch, vol. VII, p. 497 sub "palea».

2 Nous tenons ici à remercier le Père G. Gasca Queirazza de Turin qui nous a transmis, pour l'Italie du nord, une vaste documentation remontant jusqu'au XIV ${ }^{\mathbf{e}}$ siècle. Le Glossaire romand, qui a également mis ses matériaux à notre disposition, nous donne une première attestation du terme en 1570 à Sion.

${ }^{3}$ Les termes techniques cités entre guillemets appartiennent au français régional du Valais central. Les faits que nous énumérons, notamment le manque complet d'asepsie, trouvent confirmation dans l'excellent travail de Carl Müller, Volksmedizinisch-geburtshilfliche Aufzeichnungen aus dem Lötschental (Berner Beiträge zur Geschichte der Medizin und der Naturwissenschaften 3), 1969.

${ }^{4}$ Cf. Nicole Belmont, Rites et coutumes de la naissance dans le monde occidental, in La Vie medicale, Paris 1963, $\mathrm{n}^{\circ}$ de décembre, p. 104.

${ }^{5}$ Cf. Ursula Brunold-Bigler, Die Rose von Jericho (Anastatica Hierochuntiaca), eine weihnächtliche Orakelpflanze, in Schweiz. Archiv für Volkskunde n 73 (1977), p. 121-126.

${ }^{6}$ Cf. C. Müller, op. cit.

${ }^{7}$ Heinrich Schmid, Volksmedizinisches aus dem Kanton Glarus, Diss. Zürich 1924. 
${ }^{8}$ Cf. par ex. Jacques Gélis, L'accouchement au XVIII ${ }^{\mathrm{e}}$ siècle. Pratiques traditionnelles et contrôle médical, in Ethnologie française VI (1976), p. 325-340, et Marc Leproux, Du berceau à la tombe. Contributions au folklore charentais, Paris, PUF, 1959, p. 15.

${ }^{9}$ Le Musée rhétique à Coire en possède un exemplaire. A Sierre on nous a montré une «chaise obstétricale» provenant de Salquenen (Valais) où elle aurait été en usage jusqu'à la première guerre mondiale. Un baquet placé devant, rempli d'eau chaude, aurait facilité par la vapeur, «l'ouverture du passage». En fait, il s'agit d'une chaise du $\mathrm{XVIII}^{\mathrm{e}}$ siècle, ordinaire, non percée, non échancrée, mais munie de solides accoudoirs qui, si elle a vraiment été utilisée comme chaise obstétricale, a dû faire concurrence de commodité à nos bancs disposés en angle.

${ }^{10}$ Eugène Olivier, Médecine et santé dans le Pays de Vaud au XVIII ${ }^{e}$ siècle, tome premier, p. $297 \mathrm{~s}$.

${ }^{11}$ Les préparations d'ergot faisaient partie de la trousse de sage-femme qu'on remettait aux élèves sage-femme à la fin de leur cours de formation, et ce jusque dans notre siècle. Cf. aussi Marcelle Bouteiller, Médecine populaire d'hier et d'aujourd'hui, Paris 1966, p. 232.

12 Nicole Belmont, Les signes de la naissance, Paris, Plon 1971, p. 131.

Mentionnons encore que nous avons tiré profit de la consultation de:

Françoise Loux, Pratiques traditionnelles et pratiques modernes d'hygiène et de prévention de la maladie chez les mères et leurs enfants, Document de travail Cordes, Paris 1975, 209 p., et Marcelle Bouteiller, Rites et croyances de la naissance et de l'accouchement dans les provinces traditionnelles françaises, in La Vie médicale, Paris 1963, n de décembre, p. 87 à 89.

\section{Summary}

In the Roman dialects of the central Alps a word derived from the Latin word palea $=$ straw means a woman in childbed. Anthropological inquiries carried out during many years in the middle Wallis allow to point out the role of the straw in the different positions of the birth giving woman: kneeling, standing, cowering down, etc.

Mme Rose-Claire Schüle

Ethnologue aux Archives cantonales du Valais

chalet Combattion

CH-3963 Crans-sur-Sierre 